\title{
Effect of Strong External Turbulence on a Wall Jet Boundary Layer
}

\author{
Y. S. Tsai • J. C. R. Hunt • F. T. M. Nieuwstadt • \\ J. Westerweel • B. P. N. Gunasekaran
}

Received: 25 May 2006 / Accepted: 17 April 2007 /

Published online: 30 May 2007

(C) Springer Science + Business Media B.V. 2007

\begin{abstract}
The initial stage of the development of a wall jet under the influence of strong external turbulence has been studied in a novel shear-flow mixing-box experiment. A fully developed channel flow of depth $h(40 \mathrm{~mm})$ enters along the top wall of a cuboidal box of height $11 h$ in which a combination of oscillatory and turbulent velocity fluctuations are generated by a vertical oscillating grid at the midplane $5 h$ below the wall. When the ratio of the rms grid-generated velocity fluctuations, $u_{g}^{\prime}$, to the local mean velocity inside the wall jet layer, $u$, is greater than about 0.1 , significant changes are observed in the mean shear profile and in the eddy structure of the wall jet. The wall jet thickness increases by approximately $25 \%$ but the maximum velocity decreases by less than $10 \%$ compared to the case without the external turbulence. Fluctuations of the streamwise velocity component
\end{abstract}

F. T. M. Nieuwstadt: Rest in peace (1946-2005).

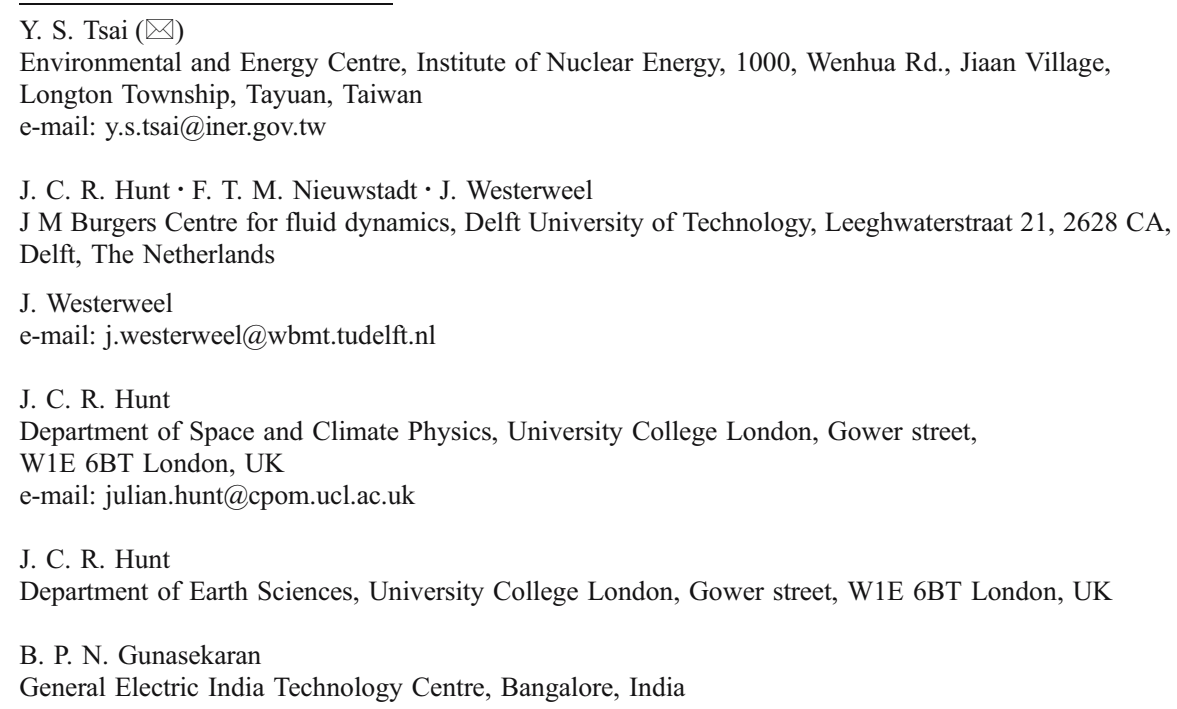


increase as expected in the outer part of the wall jet, but the most significant result is the increase by $70 \%$ of the fluctuations in the boundary layer close to the wall. CFD simulations using the $k-\varepsilon$ RNG of the FLUENT CFD Code do not properly model the effect of the large scale external turbulence in this experiment. However, an artificial method, which introduces a series of small inlet/outlet jets to represent external turbulence, approximately simulates the overall effects of the oscillating grid on the wall jet, but does not simulate the amplification of the near wall turbulence.

Keywords External turbulence $\cdot$ Wall jet $\cdot$ Mixing box $\cdot$ FLUENT simulation

\section{Introduction}

Understanding and modeling the effects of free-stream turbulence on turbulent flows near rigid walls is needed to improve the design of many types of fluid flow machinery and processes. These effects cause changes in heat transfer, particle transport, friction coefficient, and noise production on the surface. It is likely that the increase of the transfer coefficient is due to the mechanism of the interaction between the outer turbulence and the flow inside the boundary layer, resulting in the modifications of the velocity profile as well as the turbulent structure $[1,2]$. The interactions only occur when the intensity of the external turbulence is above some critical level when the eddy structures begin to change $[3,4]$. When these effects reach the region near the wall, stronger turbulence is generated within the boundary layer, as was first proposed by Taylor [5] and was recently computed using DNS by Wu et al. [6]. CFD models based on Reynolds stress closure models are not generally suitable for calculating these types of turbulent flows when the length scale of the external turbulence is relatively large compared with the shear layer thickness [7].

Experiments have shown that the influence of different levels of free stream turbulence on the mean velocity profile is primarily to suppress the wake region in the outer part of the layer while the form of the logarithmic and laminar sublayer region remains unchanged [812]. At the same time external large-scale turbulence can effectively increase the turbulent intensity close to the wall [13].

In previous studies, turbulence was conventionally generated by fixed (passive) grids. The turbulent intensity was able to reach only about $10 \%$. Although active grids (e.g. Mydlarski and Warhaft [14]) can increase the intensity of turbulence but this method is difficult to raise the level above $20 \%$ and cannot produce low frequencies with large scale eddies which are representative of many industrial and environmental turbulent flows. Another disadvantage is that the free-stream turbulence decays exponentially with the distance from the grid upstream. This affects how the boundary layer interacts with the external turbulence. Therefore, to study the effect of high intensity free stream turbulence without such decay in the flow direction, a new kind of experimental apparatus is needed. In a new design, a side-channel is used to introduce the water flow into the top of a mixing box in which there is a vertical oscillating grid well below the channel to provide strong free-stream turbulence. This design, based on the hybrid system for the mixing process of a shear flow and grid-generated turbulence, was first introduced by Thomas and Simpson [15] to study how the external turbulence influences the mixing of a saline gravity current on a fresh water flow with opposite direction.

In the present setup, the streaming shear flow directed along the wall enters the larger volume of the mixing box. A plane wall jet forms for which the velocity profile contains 
two distinct flow regions. The inner flow, which lies between the surface and the point of the maximum velocity, is broadly similar to a boundary layer. The outer part away from the maximum is like a free shear layer. Some upstream effects in the entry channel may be induced by the interactions between the wall jet and the grid induced fluctuations.

In the measurement of wall jets, it has been observed that the mean velocity and turbulence profiles are self-similar over long distances downstream of the initiation of the jets [16-18]. Conventional scaling parameters are the mean maximum velocity, $u_{\max }$, and the upper half-width, $y_{1 / 2}^{U}$, where the mean velocity is equal to one half of the maximum velocity for the outer layer, and the friction velocity $u_{\tau}$ and the kinematic viscosity $v$ for the inner layer. More recently, Barenblatt et al. [19] suggests that the lower half-width, $y_{1 / 2}^{L}$, is also an important scaling parameter to present the incomplete similarity of the turbulent wall jet. Hence three layers of the velocity profile, top, intermediate, and wall layer are divided by $y_{1 / 2}^{U}$ and $y_{1 / 2}^{L}$. The aim of this experiment is to study the effect of high intensity external turbulence on the wall jet by considering the changes of the velocity and the turbulent intensity profile both in the boundary and in the free shear layer.

Although on the basis of previous experiments external turbulence can have a significant effects on turbulent shear layers, detailed studies are needed to understand how intense external turbulence, such as that generated by a vertical oscillating grid, leads to changes within a wall jet. When the external turbulence is weak, the shear layer 'shelters' the boundary layer from the external turbulence [3]. However, when it is sufficiently strong, for example, $u_{g}^{\prime} / u$ greater than the order of 0.1 (where $u_{g}^{\prime}$ denotes the oscillating gridgenerated turbulence and $u$ denotes the local mean velocity inside the boundary layer), then the shear layer is strongly affected and spreads outwards. It is also necessary to understand that the shear layer consists of growing vortices and that the turbulence produced by the oscillating grid contains vortices. As these two sets of vortices interact, chaotic merging and expulsion of vortices may occur [20]. Also, the increased intensity of turbulence in the free shear layer affects the turbulence in the boundary layer near the wall $[10,21]$. The present experiment is designed to study all these interactions. This indirect effect of external turbulence in wall layer has not been studied previously and it has direct relevance in studying turbulence modification near the wall and transport process in many types of complex flows.

\section{Experimental Arrangement}

\subsection{Test facility}

The present experimental apparatus is shown in Fig. 1. The coordinate origin is located at the surface of the contact plane of the inlet channel and the mixing box. $\mathrm{X}$ is measured in the flow direction and $\mathrm{Y}$ is measured downwards from the surface. A pump drives the water through the circuit with a maximum flow rate of $3.1 \times 10^{-3} \mathrm{~m}^{3} / \mathrm{s}$. A diffuser is used to smooth the transition between a short length of pipe from the reservoir to the channel flow that enters the mixing box. Four guide vanes and seven layer monoplane meshes are inserted in the diffuser to prevent any flow separation from the side walls and also to generate a uniform flow. The dimensions of the channel are $530 \mathrm{~mm}$ in length, $350 \mathrm{~mm}$ in width, and $h=40 \mathrm{~mm}$ in height. Its side-walls and upper container wall join smoothly to the side walls and top wall $(y=0)$ of the mixing box.

In order to produce a fully developed channel flow in the present short distance of the inlet channel, two rubber strips $3 \mathrm{~mm}$ in height and $20 \mathrm{~mm}$ in length are placed spanwise 


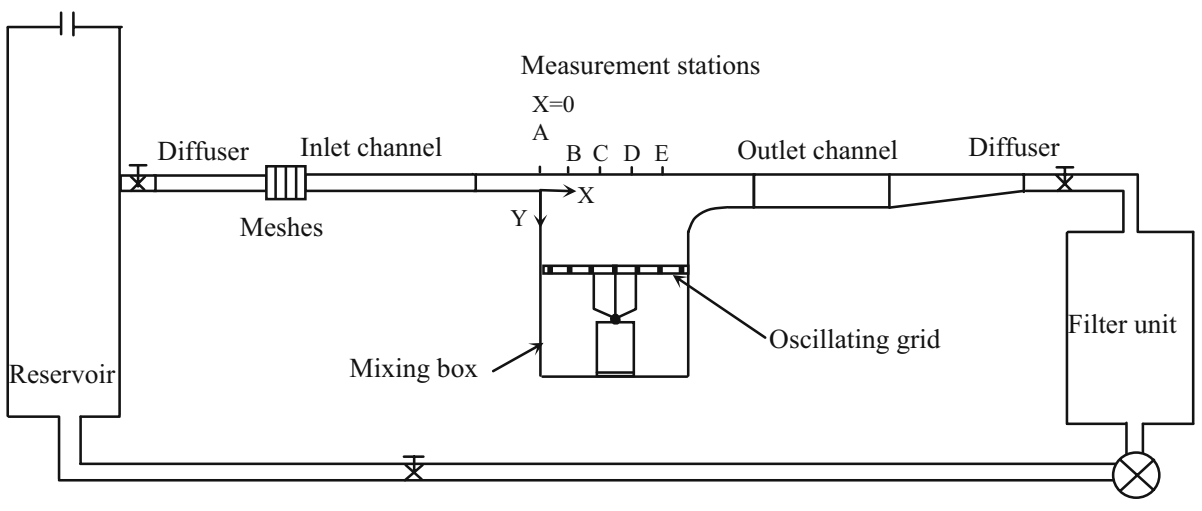

Pump

Fig. 1 Schematic of the hybrid experimental setup. The inlet channel height was $40 \mathrm{~mm}$ in height and the size of the rectangular mixing box was $350 \times 350 \mathrm{~mm}^{2}$ with a $540 \mathrm{~mm}$ in height

across the channel on both the upper and lower walls. They are located immediately behind the mesh chamber to trigger the turbulent boundary layer.

There is a sharp right angle at the connection point of the lower channel wall and the mixing box, which initiates the wall jet. The width to the height ratio is $w / h=7.5$. The cuboidal mixing box $(350 \times 350 \mathrm{~mm}$ and $540 \mathrm{~mm}$ in height $)$ contains the oscillating grid located at the level $y=5 h$ to produce velocity fluctuations which are transported by the turbulent eddies to the wall jet. Both the channel and mixing box were made of transparent polyvinyl chloride to facilitate optical measurements and flow visualizations. The size of the monoplane grid is $50 \times 50 \mathrm{~mm}$ and the width of the grid bars is $1 \mathrm{~cm}$. The grid has a proper end condition, that is, the distance between the wall and the closest bar parallel to the wall is $M / 2$ where $M$ is the mesh size. With this design, the grid generated large-scale circulation can be reduced [22]. Accordingly, the solidity is $40 \%$. The grid is connected to a pneumatically driven actuator, which oscillates vertically in a step up/down motion. The frequency is computer controlled. The external velocity fluctuations (rms value $u_{g}^{\prime}$ ) generated by the mixing jets and wakes behind the grid bars are a combination of an oscillatory component $u_{g o}^{\prime}$, and turbulence $u_{g t}^{\prime}$. The triple decomposition analysis shows that the oscillatory component in the streamwise direction is insignificant. This component may have significant effect in vertical (wall-normal) direction.

The grid oscillated at 1 and $3 \mathrm{~Hz}$ with a stroke of 50 and $43 \mathrm{~mm}$, respectively, for the present measurements. This gave a different levels of rms turbulent fluctuations, $u_{g t}^{\prime}$. Hopfinger and Toly [23] quantified the turbulence generated by an oscillating grid as follows:

$$
u_{g t}^{\prime}=C S^{3 / 2} M^{1 / 2} f_{g}\left(y-y_{o}\right)^{-1}
$$

where $C$ is a coefficient ( $C \approx 0.25$ in previous mixing box experiments), $S$ the stroke, $M$ the mesh size, $f_{g}$ the oscillating frequency, and $y_{o}$ the virtual origin which is close to the midplane of the grid. From (1), the grid turbulence at $3 \mathrm{~Hz}$ was 2.4 times greater when compared with that at $1 \mathrm{~Hz}$ at the same vertical distance from the grid.

For the present experimental setup with a shear flow growing in the upper part of the box, a large-scale circulation in the mixing box is driven by the entrainment into the upstream wall jet layer and by the flow impingement of the jet on the downstream wall. A CFD simulation using FLUENT 6.0 code was employed to help the design of the mixing 
box geometry in order to estimate the magnitude of the circulation and to reduce it. The simulation revealed that when the outlet channel had the same height as the inlet channel, the jet impingement on the wall induced a strong circulation as well as a high level of turbulence in the downstream part of the box. To address this problem, the flow outlet channel was varied with an initially curved floor connecting the downstream wall of the box to the outlet channel floor. The outlet channel downstream was double the inlet channel height. This design effectively reduced the upstream effects of the flow impingement on the downstream wall. The CFD simulation showed that the main-stream velocity decreased smoothly in this adjustment region. The re-circulation velocity in the mixing box was predicted to be approximately $10 \%$ of the main stream velocity. Besides, the turbulence intensity inside the box was also significantly reduced.

\subsection{Flow visualization using LIF}

Experiments with laser induced fluorescence (LIF) were conducted to visualize the general flow structure in this new setup, particularly the effect of the external turbulence. To illuminate the fluorescent dye, a laser sheet with $3 \mathrm{~mm}$ in thickness generated by an Argoniron laser was vertically projected from the top cover at the middle of the spanwise width. The fluorescent dye was injected through a rigid tube with a hole of $0.5 \mathrm{~mm}$ in diameter on the side near the tube bottom, inserted into the channel $155 \mathrm{~mm}$ upstream from the origin. To observe the flow pattern in the outer mixing layer and in the inner boundary layer region surface, two vertical injection positions were chosen. They were at the bottom of the channel and $1 \mathrm{~mm}$ from the top wall, respectively. The dye spread along the channel and down into the mixing box.

\subsection{LDA velocity measurement}

The wall jet velocity profile and turbulent fluctuations in the streamwise direction were measured using Laser Doppler Anemometry (LDA) for the pure wall jet flow at four stations $\mathrm{B}(x / h=1.75), \mathrm{C}(x / h=3.5), \mathrm{D}(x / h=5.25)$, and $\mathrm{E}(x / h=7)$ and under the circumstance with grid oscillation at $1 \mathrm{~Hz}$ at three stations $\mathrm{B}, \mathrm{C}$, and D. The LDA is single component fiber optics system in backward scatter mode, powered by a $4 \mathrm{~W}$ Argonion Spectra-Physics model 2000 laser. The optical elements included a beam splitter, Bragg cell and a $600 \mathrm{~mm}$ focusing lens. The probe measuring volume, based on the $e^{-2}$ light intensity cutoff point, was $0.29 \mathrm{~mm}$ in diameter and $10.8 \mathrm{~mm}$ in length. Signal processing was performed by a Dantec Burst Spectrum Analyzer (BSA, type Enhanced 57N10) and data were collected using a LabVIEW instrument program. Eight micrometers particles were employed for seeding the flow. The sampling rate was around $200 \mathrm{~Hz}$ in the main stream. However, it decreased to about $50 \mathrm{~Hz}$ both in the near wall region and in the far outer layer due to the low speed in these two regions. The recording time for each measuring point was $180 \mathrm{~s}$. To analyse the spectrum of the turbulent fluctuations, the LDA data record was reproduced by linear interpolation between two adjacent points in the raw signal to obtain evenly spaced time steps. The time intervals were equal to the average time between the data points in the record.

The LDA probe was rigidly mounted in a three axis traversing system, controlled by a PC with a positioning precision of $0.01 \mathrm{~mm}$. Observation of the output signal in the BSA was used to define the wall position $(y=0)$, which was determined by carefully traversing the probe upwards. When the measurement volume touched the wall, a noising signal immediately occurred, judged by extraordinary large numbers of bursts shown on the BSA. 
With this method, the closest measurement point was located approximately $0.2 \mathrm{~mm}$ from the surface. Given the low flow speed of this experiment, this distance was inside the laminar sublayer.

For LDA measurement the velocity is generally biased to a higher value. The difference increases with the increase of the turbulence intensity. The straightforward corrections such as one-dimensional inverse velocity weighting or weighting by interarrival time are not suitable to apply here $[24,25]$, because the present complex turbulent flow with the external turbulence was unsteady three dimensional flow and was measured with limited data rate. For this reason, the velocity was not corrected in the present study. The worst condition occurs for the highest turbulence level at station $\mathrm{D}$ in the upper layer with the gridgenerated turbulence was estimated to be approximately $8 \%$ for mean velocity and $5 \%$ for the root mean square of the turbulent fluctuations [26]. Nevertheless, the measurement showed that the flow pattern changed by the external turbulent fluctuations was significantly greater than the LDA errors. The velocity gradient effect across the measurement volume was corrected using the formula given by Drust et al. [27].

\section{Experimental Result}

3.1 Mean flow and turbulence in the inlet

The inlet condition for the formation of the wall jet was made at the contact plane of the inlet channel and the mixing box ( $x=0$, position A in Fig. 1). The flow speed was $0.11 \mathrm{~m} / \mathrm{s}$ which corresponded to the Reynolds number 2200, based on half of the channel height. The LDA measurements show that the velocity variation in the spanwise direction was less than $\pm 0.8 \%$. This profile defines the plane wall jet as the flow enters the channel. Figure 2 displays the mean velocity profile and turbulent intensity with both the measurement and FLUENT simulation using a RNG $k-\varepsilon$ model. The present measurement of the velocity
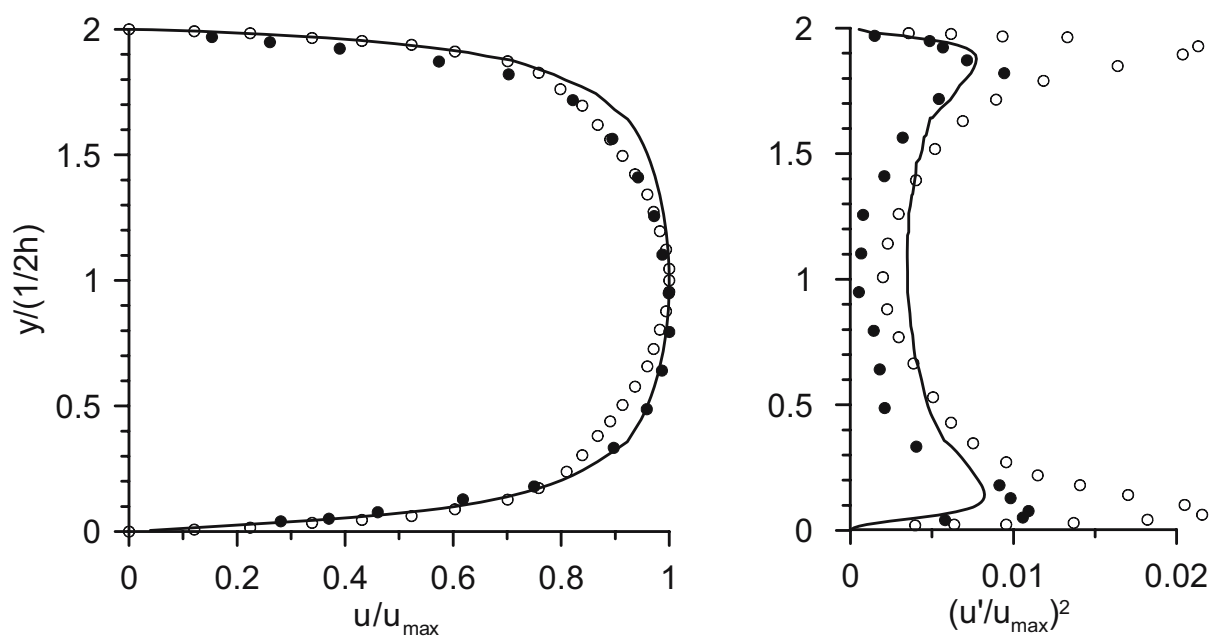

Fig. 2 The inlet condition of the mean velocity and turbulence intensity profiles at $x=0$ (position A). Filled circle, present measurement; lines, FLUENT simulation; $R e=2200$; open circle, Niederschulte et al. [28], $R e=3300$ 
profile is close to the observation by Niederschulte et al. [28] for a fully developed channel flow. However, the actual profile may not have been fully developed and was likely to be developing in the FLUENT simulation. The turbulent intensity is significant greater in Niederschulte et al. [28]. This may be due to a higher Reynolds number in their experiments. Simulation shows a higher turbulence level in the middle of the channel than the measurements of $u^{\prime}$, which are estimated from the turbulent energy $k$ with $k=3 / 2\left(u^{\prime}\right)^{2}$.

\subsection{Flow structure in the mixing box}

The visualization shows how the flow in the inlet channel enters into the mixing box and forms a free shear layer. It also reveals how the inlet channel flow impinges on the downstream wall and forms a weak circulation in the box. It is estimated from the recorded video that the circulation is approximately $5 \%$ of the main stream velocity. This is significantly smaller than the prediction from the FLUENT results perhaps because the code assumes high Reynolds number turbulence and therefore higher entrainment into the free shear layer. Thus, the external circulation is weak and any side-effect on the shear stream is limited.

Figure 3 demonstrates a portion of the flow structures in the outer layer with and without the grid-generated turbulence over a distance between 0.5 and $7.5 \mathrm{~h}$ in streamwise direction. From the flow visualization, for the case without the external turbulence in Fig. 3a,b, largescale vortices were observed within the shear layer. They grow with downstream distance and hence their spacing increases. The observed frequency $f_{v}$ is approximately $0.92 \mathrm{~Hz}$ at $x \approx 0.5 h$ with the wave length $\lambda_{v}=u / f_{v} h=1.1$ and the Strouhal number $S_{v}=f_{v} h / u_{\max }=0.33$. The vortices had irregular shapes due to the effect of internal turbulence arising from the turbulent channel flow. The large-scale vortices progressively break down into small-scale eddies along the flow (Fig. 3b). When the grid oscillated at $1 \mathrm{~Hz}$ so that $S_{g}=0.36$ (approximately equal to $S_{v}$ ) and $u_{g}^{\prime} / u=0.15$ at the level of the channel depth $\left(u_{g}^{\prime}\right.$ is calculated from (1) with $C=0.45$ ), the interaction between the external turbulence and the mixing stream significantly increases the diameter of the large-scale vortices in the shear layer. However, the rotation speed of the vortices decreases which suggests that the circulation of the vortices is approximately conserved. The wavelength between the vortices $\lambda_{v}$ increases by a factor of three. At the same time, vortices from the shear layer burst intermittently upwards to the surface layer (arrow mark in Fig. 3c). This process increases the thickness of the mixing layer and diminishes the maximum kinetic energy of the largescale eddies. The phenomenon shows how the external turbulence triggers the transport of eddies from the outer mixing layer to the wall boundary layer. At a higher oscillation frequency of the grid at $3 \mathrm{~Hz}\left(S_{g}=1.08\right)$, the external turbulence $\left(u_{g}^{\prime} / u=0.36\right)$ was further increased. The mixing processes are similar to those with the grid oscillating at $1 \mathrm{~Hz}$. However, the large-scale vortices were further weakened with more frequent occurrences of the 'bursting', leading to a rapid mixing process. Generally, the velocity fluctuations through the whole layer are increased by the oscillating grid. The resulting characteristic flow pattern in the outer layer consist of large-eddies, the occurrence of bursting phenomena, and the increased width of the shear layer at the edge of the wall jet.

Figure 4 shows how the external fluctuations affect the inner boundary layer. Without the grid turbulence, the flow structure near the wall is composed of large bulges with hairpin vortices (Fig. 4a,b), as in a turbulent boundary layer developed on a rigid flat plate at low Reynolds number [29]. In the presence of external turbulence generated by the grid, there is an increase of the irregularity of the flow pattern and intensity of the turbulence 


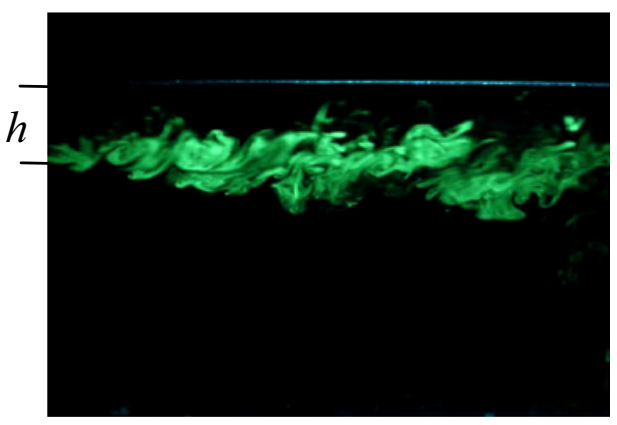

a

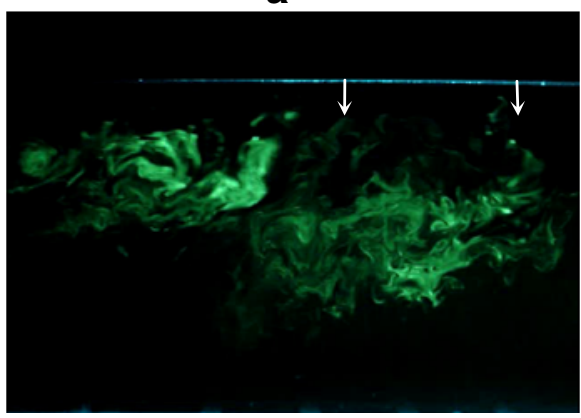

C

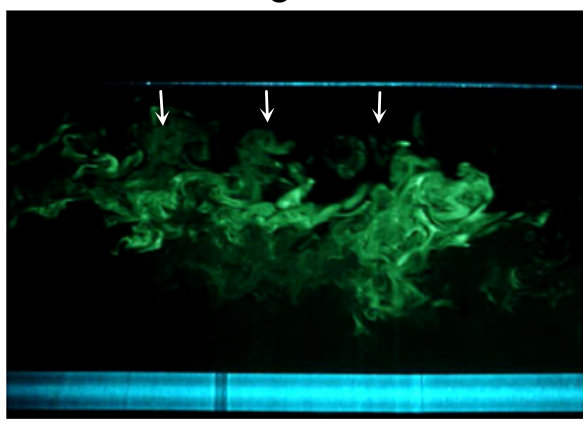

e

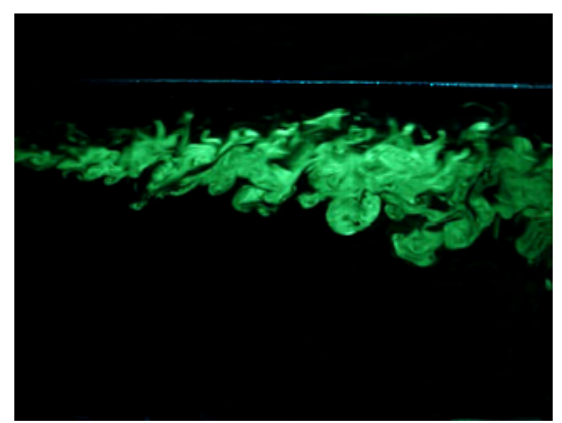

b

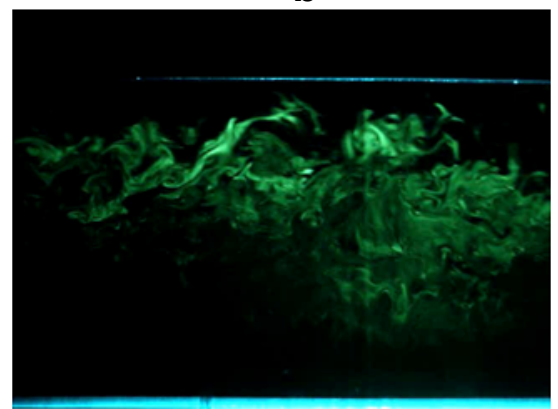

d

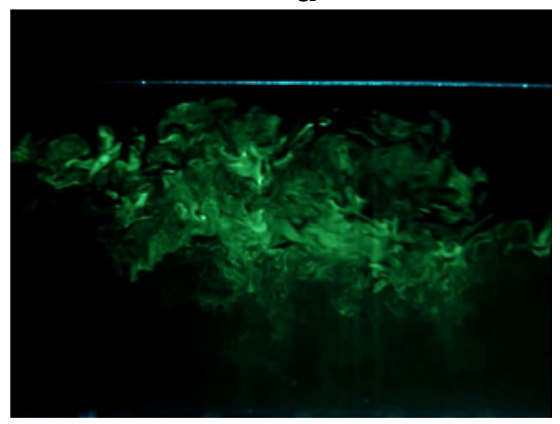

f $7.5 h$ $0.5 \mathrm{~h}$

Fig. 3 Flow pattern visualized by fluorescence in the outer mixing layer region a and $\mathbf{b}$ without gridgenerated external velocity fluctuations; $\mathbf{c}$ and $\mathbf{d}$ grid oscillated at $1 \mathrm{~Hz}$; e and $\mathbf{f}$ grid oscillated at $3 \mathrm{~Hz}$. Injection position: bottom of the channel, $155 \mathrm{~mm}$ upstream. The illuminated bar in (e) is the oscillating grid. The flow conditions are $u_{\max }=0.11 \mathrm{~m} / \mathrm{s} ; R e=2200 ; u_{g}^{\prime} / u \approx 0.15$ and 0.36 for 1 and $3 \mathrm{~Hz}$, respectively, at the depth of the channel height. Arrows indicate the bursting phenomena stimulated by external fluctuations

inside the boundary layer. The bulges significantly grow with downstream distance and the flow pattern shows that the vertical velocity fluctuations transport fluid from the inner region to the outer mixing layer. The observation shows that the external turbulence affects the near wall region by a different mechanism. This involves the interaction between the near wall bulges and shear layer eddies, which is primarily enhanced by the external turbulence. 


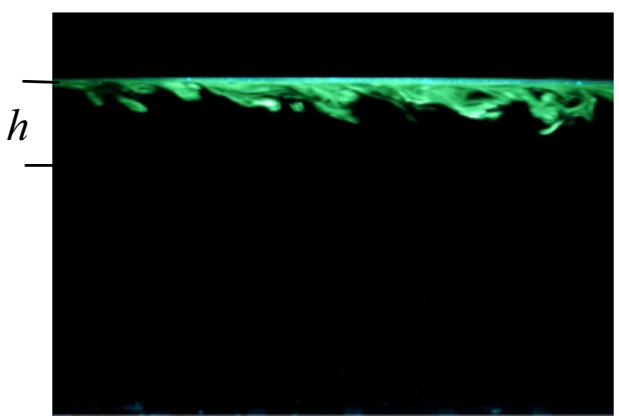

a

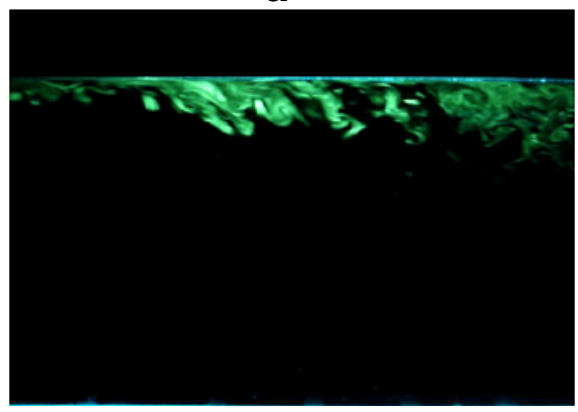

C

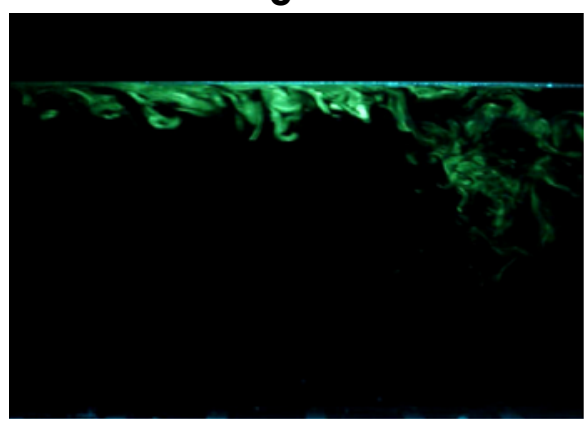

e

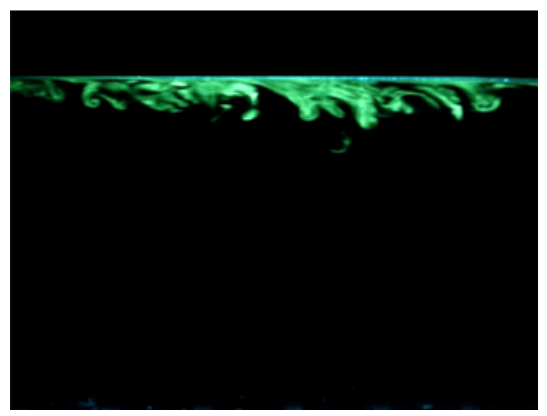

b

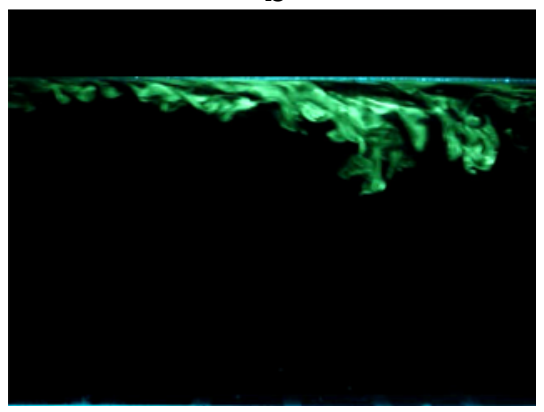

d

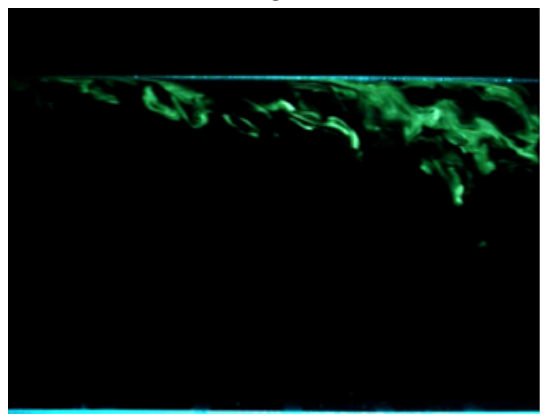

f

Fig. 4 Flow pattern visualized by fluorescence in the upper near wall region $\mathbf{a}$ and $\mathbf{b}$ without grid generated turbulence; $\mathbf{c}$ and $\mathbf{d}$ grid oscillated at $1 \mathrm{~Hz}$; e and $\mathbf{f}$ grid oscillated at $3 \mathrm{~Hz}$. Injection position: $1 \mathrm{~mm}$ from the top wall

\subsection{Wall jet mean velocity and turbulence profile}

The measurements of the mean velocity profiles in the wall jet are shown in Fig. 5 both with and without the external fluctuations. The wall jets grow in thickness and their maximum velocities decrease with downstream distance. In particular, the high external velocity fluctuations transport the mean momentum of the jet outwards into the mixing layer. The wall jet thickness increases rapidly and the maximum velocity decreases more rapidly in the downstream distance than in a pure wall jet development. The same mechanism causes the location of the maximum velocity, $y_{m}$, to be driven further away from the wall. However external turbulence can increase local transport near the wall. Consequently in the near wall region, the external turbulence slightly reduces the gradient 


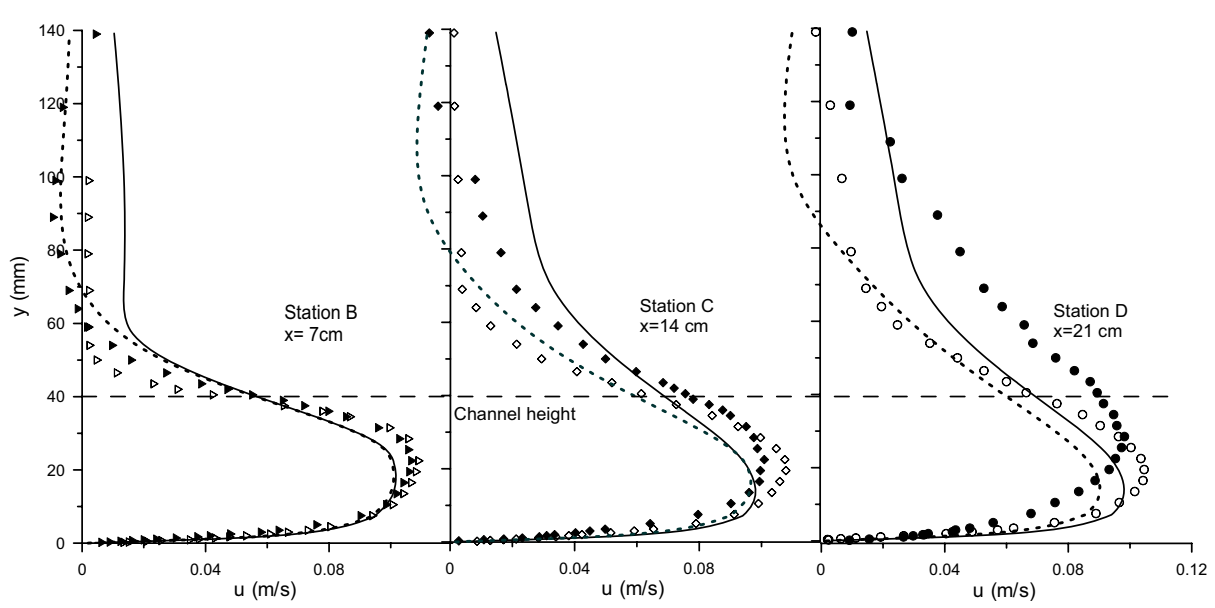

Fig. 5 Experiments and simulations of the mean velocity of the wall jet development with and without the external velocity fluctuations. Experiment: open right triangle, open diamond, open circle, without oscillating grid; filled right triangle, filled diamond, filled circle with oscillating grid. FLUENT simulation: dashed lines, without the external turbulence; dotted lines, with the external turbulence, simulated by inlet/ outlet jets

of the velocity profile and the friction on the wall is also slightly decreased. This is in contradiction to the previous studies of Simonich and Bradshaw [1] and Blair [8,9] which show how free-stream turbulence increases the skin friction on a flat plate. This may be because of the large scale oscillatory nature of the type of velocity fluctuations in this experiment.

The non-dimensional velocity profiles using both the upper and lower half width $y_{1 / 2}^{U}$ and $y_{1 / 2}^{L}$ and the maximum velocity $u_{\max }$ are plotted in Fig. 6 . In all three stations the profiles show self-similarity in the top and intermediate layer $\left(y / y_{1 / 2}^{L}>1\right)$ when scaled by $y_{1 / 2}^{U}$ under different free-stream turbulence. Negative velocities in the outer layer at station B show that the grid oscillation increases the circulation area. For the region close to the wall $\left(y / y_{1 / 2}^{L}<1\right)$ using the inner parameter $y_{1 / 2}^{L}$, with the external turbulence the velocity is higher than that without external turbulence at station $\mathrm{B}$, but the two profiles collapse at station $\mathrm{C}$ and have an opposite position at station D. Similarity occurs at station C but not in station B and D.

Figure 7 shows the non-dimensional velocity profiles using the inner parameters $u^{+}=$ $u / u_{\tau}$ and $y^{+}=u_{\tau} y / v$. The friction velocities were obtained using a straight line $y^{+}=u^{+}$ fitted to the measurement points near the wall $y^{+}<5$. In previous study, it has been observed that on a rigid flat surface the free-stream turbulence diminishes the boundary layer wake region and the length of the logarithmic region is increased $[8,9,11,12]$. For the present wall jet, the location of the maximum velocity $y_{m}^{+}$at station B with the external fluctuations effect has a smaller value than that without the external velocity fluctuations. However, the values are approximately the same at station $\mathrm{C}$, but the trend is reversed at station $\mathrm{D}$. The logarithmic region does not consistently increase as observed with the external turbulence on a flat plate. The experiments of Tachie et al. [18] shows a self-similarity both in the laminar sublayer and logarithmic region for a fully developed wall jet in the measurement distance between $30<x / b<80$, where $\mathrm{b}$ is the height of the slot. The present data for $y^{+}<4$ is likely to be similar with or without external velocity fluctuations. However, for the logarithmic region, the profiles do not reveal a similarity. This may be because the present measurement was located in the range $x / h<5.25$ where the profile was still developing. 

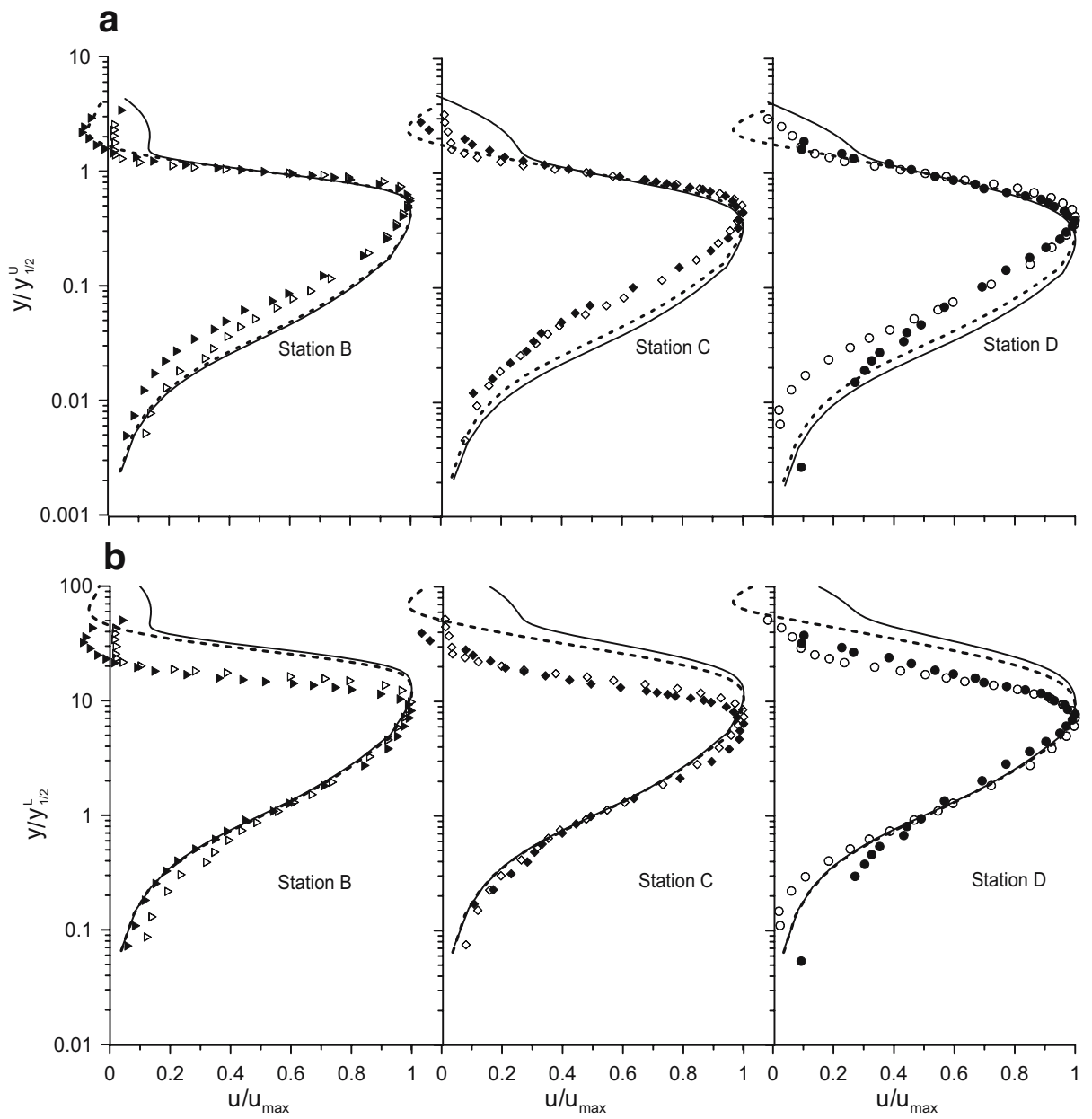

Fig. 6 Mean velocity profiles scaled using the a upper and $\mathbf{b}$ lower half-width and maximum velocity. The symbols represented are same as in Fig. 5

Barenblatt et al. [19] found that the growth of the half-width in an intermediate region downstream, $h \ll x \ll H$, where $\mathrm{H}$ was a characteristic length of the setup, depending on the height of the slot, according to the scaling law

$$
y_{1 / 2}^{i} / h=A_{i}(x / h)^{\beta_{i}}
$$

where $i=U$ denotes the upper layer and $i=L$ denotes the lower layer, representing the incomplete similarity. Figure 8 demonstrates the growth of the dimensionless half-width in the present measurements. Without the external turbulence $\beta_{U}=0.22$ and $\beta_{L}=0.2$, the difference is $10 \%$. This is not as significant as shown in Barenblatt et al's paper. With the external turbulence, $\beta_{U}$ and $\beta_{L}$ substantially increases with the values of 0.51 and 0.27 , respectively. However, except the growth of $y_{1 / 2}^{L}$ with the external turbulence, a linear fitting gives a better agreement. In this point of view, whether incomplete similarity occurs in different free-stream turbulence level can not be concluded here. 
Fig. 7 The mean velocity profiles scaled using the inner parameters

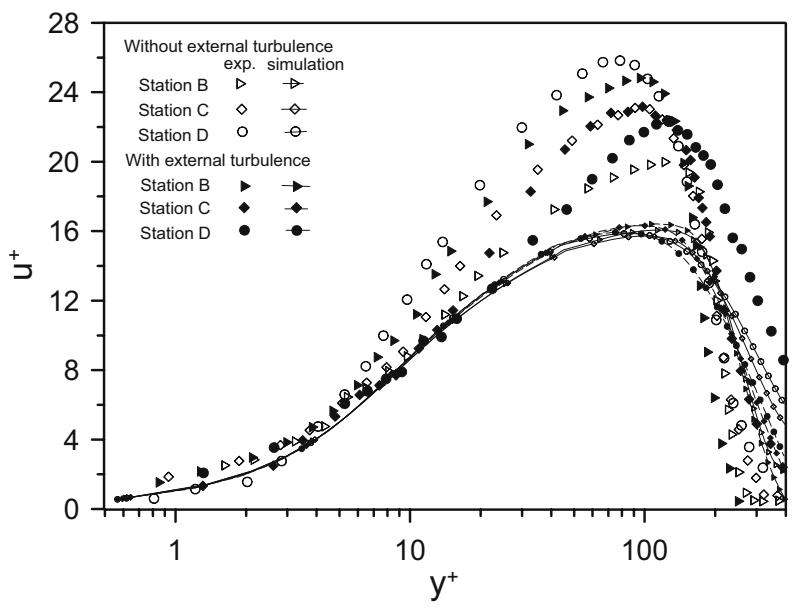

The comparison between the fluctuations of the streamwise component for the wall jet with and without the grid-generated turbulence is depicted in Fig. 9 together with the line of the pure grid-generated turbulence from (1). The measurement with only the grid oscillation in the box at station $\mathrm{C}$ shows a better agreement with formula (1) when the coefficient $\mathrm{C}$ is about 0.45 , rather than the value $C=0.25$ of Hopfinger and Toly [22], whose experiments were in a cubical box. With the grid turbulence and the shear flow, the conspicuous features of the fluctuation profiles are that there are two local peaks. One is in the outer mixing layer, denoted as P1, which is caused by the interfacial disturbances at the position around the channel depth. Another occurs in the inner boundary layer region in the vicinity of viscous sublayer, denoted as P2. The effect of the external fluctuations is to increase the level of fluctuations throughout the whole wall jet. The largest relative increase is observed in the region below the channel depth $y=h$ where the flow only consist of the low speed large-scale circulation and the grid turbulence. The maximum value P1 in the mixing region moves downwards as the thickness of the mixing layer increase. With the high level of fluctuations turbulence generated by the oscillating grid, the turbulent energy is increased in

Fig. 8 Growth of the upper and lower half width with respect to distance

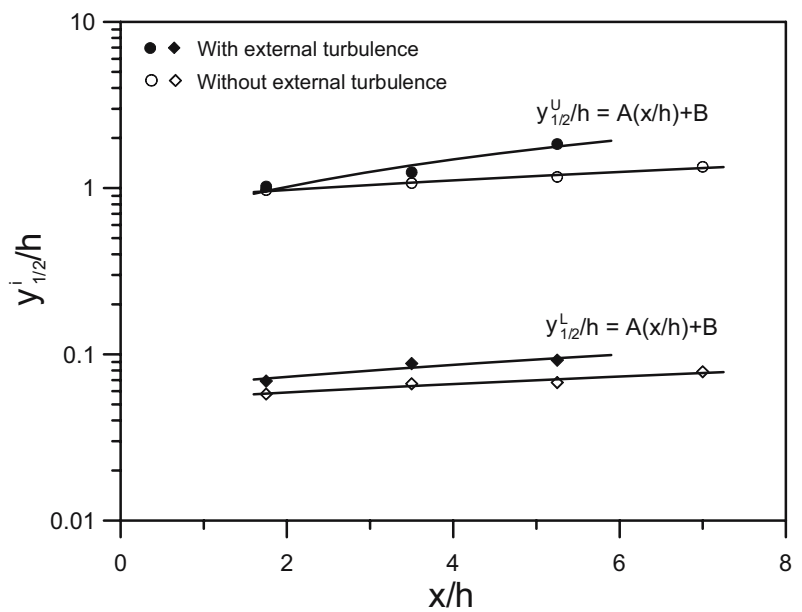




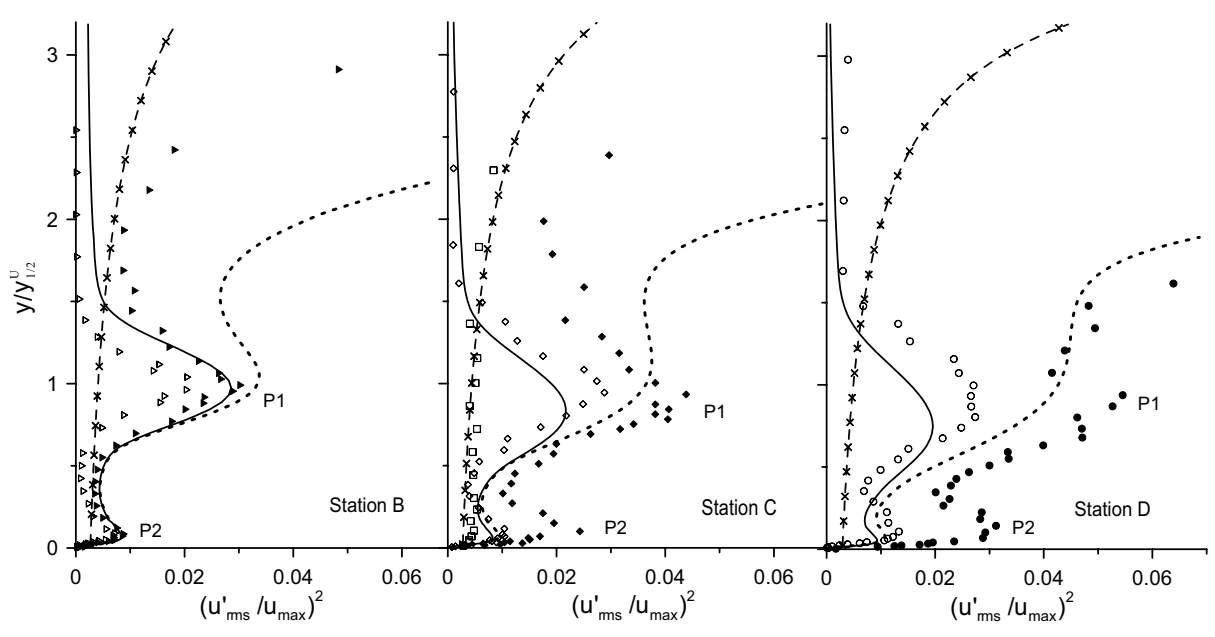

Fig. 9 Experiments and simulations of the energy of the streamwise velocity fluctuations scaled using the outer parameters with and without the external turbulence. Pure oscillating grid: square, experiment; hyphens with an $x$ at the center, theory from (1); Other symbols and lines are defined in Fig. 5

the near wall region. As the visualization shows eddies penetrate the mixing sheltering shear layer and amplify the peak of P2. The peak increases along with the downstream distance. Significant and continuous increase of the turbulent energy can be found at stations $\mathrm{C}$ and $\mathrm{D}$. The LDA measurement and the LIF visualization for the same flows as in Fig. 4c,d both show that the flow pattern becomes irregular and that hairpin vortices grow spectacularly.

Figure 10 presents the streamwise fluctuation profiles using the inner parameters. In the region $y^{+}<100$, both with and without the grid-generated turbulence, the turbulence intensity increases with downstream distance. At each station, the external fluctuations substantially enhance the transport of turbulent energy downwards to the laminar sublayer. A likely mechanism for the amplification of the near wall turbulence is the interaction of the increased outer mixing layer turbulence with the inner mean shear [6].

Fig. 10 Variance of the streamwise velocity fluctuations scaled plotted using wall friction parameters

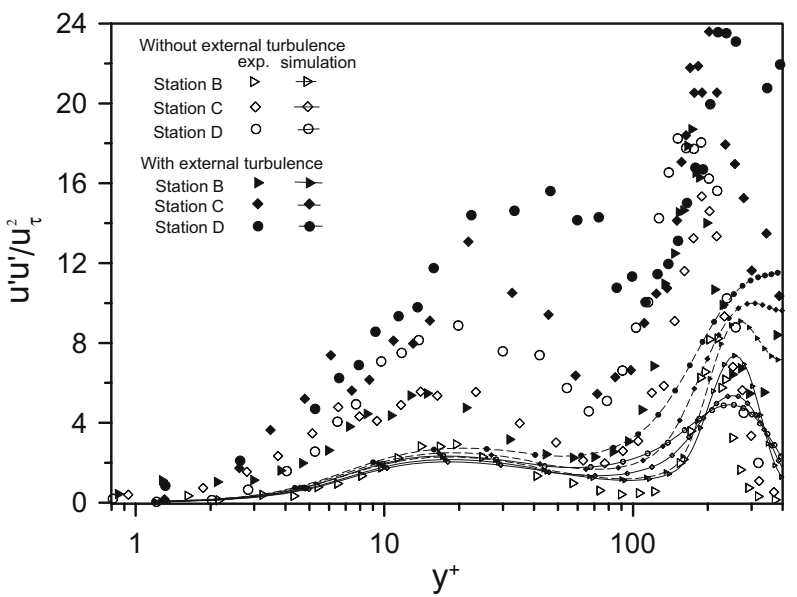

Springer 
As shown in the LIF visualization, bursting events inject energy to the near wall flow with large hairpin structures.

\subsection{Spectral analysis}

Spectral analysis of the velocity fluctuations reveals different aspects about this complex flow. The power spectrum was calculated by means of the FFT algorithm and a Hanning window with ensemble average over 30 data sets. Figure 11 demonstrates the nondimensionalized form of the spectrum using the inner parameters. Three downstream stations and three vertical positions, which are close to the wall region $y^{+} \approx 10$, in the inner

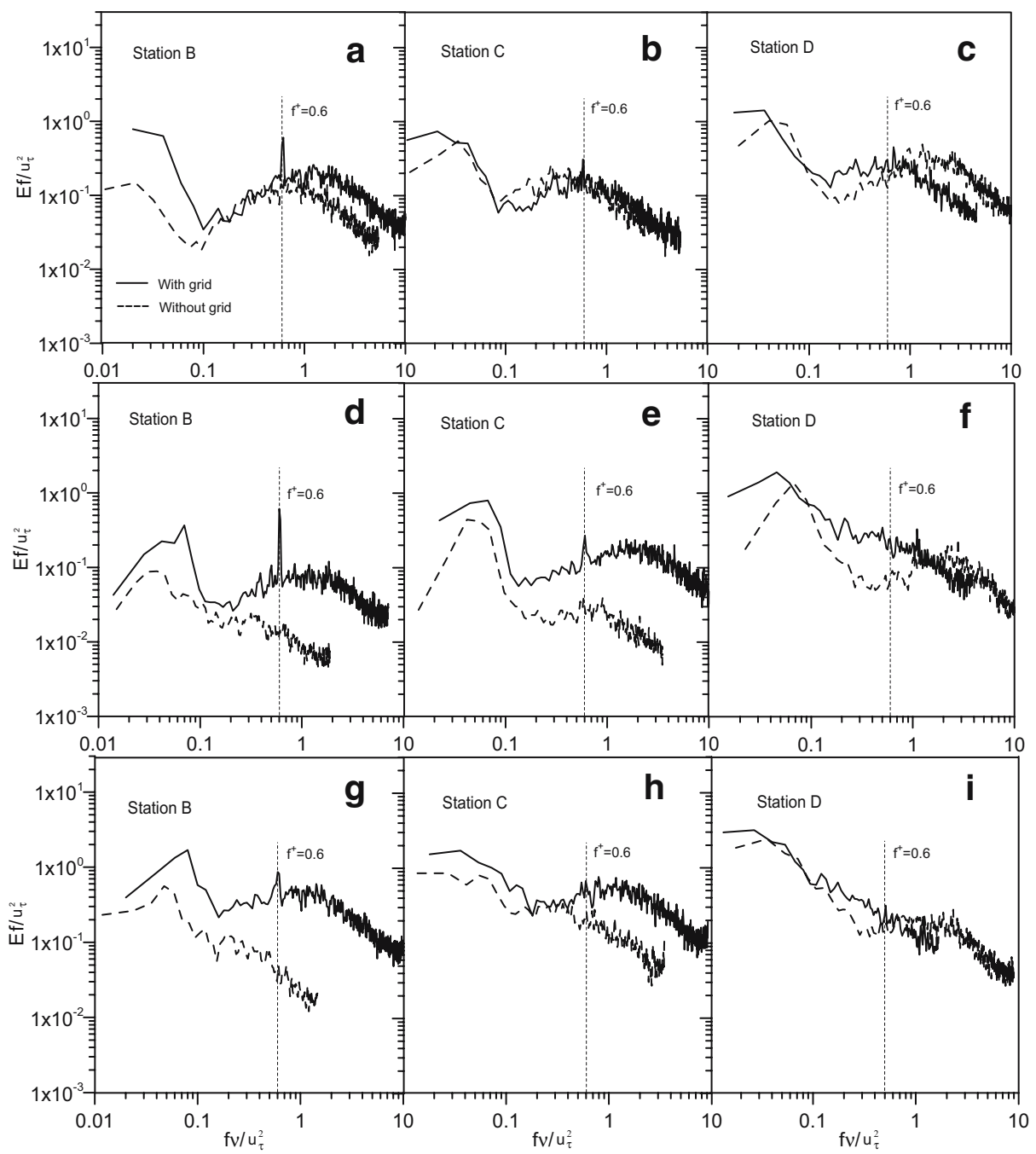

Fig. 11 Power spectrum of the streamwise fluctuations plotted in $\log -\log$ coordinates with and without the external velocity fluctuations. $\mathbf{a}, \mathbf{b}$, and $\mathbf{c}$ are in the region close to the wall, $y^{+} \approx 10 ; \mathbf{d}$, e, and $\mathbf{f}$ are in the inner boundary region, $y / y_{1 / 2}^{U} \approx 0.4 ; \mathbf{g}, \mathbf{h}$, and $\mathbf{i}$ are in the outer mixing region $y / y_{1 / 2}^{U} \approx 1.2, f^{+}=f \nu / u_{\tau}^{2}$ 
layer close to the maximum velocity $y_{1 / 2}^{U} \approx 0.4$, and in the outer mixing layer $y_{1 / 2}^{U} \approx 1.2$ are displayed.

For the pure wall jet without the external turbulence, at the location of $y^{+} \approx 10$, the spectrum in Fig. 11a,b, and c shows two distinct features. For the non-dimensional frequency $f^{+}<0.1$, there is a large-scale structure with the energy growing with the downstream distance. This elongated structure is caused by the low-speed streaks in the buffer layer which (in low Reynolds number turbulence) interact with the eddies throughout the shear layer [30, 31]. LIF visualization in Figs. $4 \mathrm{a}$ and $5 \mathrm{~b}$ confirms this phenomenon in a turbulent wall jet layer. In the range of $f^{+}>0.1$, the maximum is associated with near wall turbulence. At the level $y_{1 / 2}^{U} \approx 0.4$ in Fig. $11 \mathrm{~d}$,e, and $\mathrm{f}$, the large-scale eddies grow more significantly at station $\mathrm{C}$ and $\mathrm{D}$. The maximum value is located approximately at $1 \mathrm{~Hz}\left(f^{+} \approx\right.$ 0.05). The main contribution is likely to be from the vortices arising from shear layer instability as visualized in Fig. $4 \mathrm{a}$ and $\mathrm{b}$. At this distance away from the wall, the boundary layer turbulence is insignificant at stations B and C. This indicates that in this region the flow is dominated by the large-spanwise vortices with the velocity fluctuations exhibited in the low frequency part. However, as the peak broadens at station D this indicates that the large structure has broken into small-scale eddies. In the outer layer, the sharp peak in the spectrum at the initial stage B in Fig. $11 \mathrm{~g}$ is distributed over a wider range of eddy sizes through the action of the large external eddies at stations $\mathrm{C}$ and $\mathrm{D}$. The breakdown of largescale eddies transfers the energy to small-scale eddies in the high frequency range, reducing the maximum as shown in Fig. 11i.

With the grid oscillating at $1 \mathrm{~Hz}$, the grid-generated turbulence interacts with the shear layer turbulence. In general, the interaction causes the energy growth inside the large-scale eddies in the low frequency part (approximately $f^{+}<0.1$ ) of the spectrum through the whole wall jet layer and downstream stations. On the other hand, for the high frequency part, the grid turbulence generated a maximum in both the inner and outer layer at station $\mathrm{B}$ and $\mathrm{C}$ (Fig. 11d,e,g, and h), significantly increasing the energy level as compared to the condition without the external fluctuations. However, the maximum disappears at station D in Fig. $11 \mathrm{f}$ and $i$. The general trend of the external fluctuations is to produce a fully mixed region downstream. One striking phenomenon observed is that there is a second peak inside the hump at $f^{+} \approx 0.6$, which even has the maximum value in Fig. $11 \mathrm{~d}$. This reveals that resonance occurs in this particular frequency when the two turbulence streams encounter but the peak value decreases in the downstream stations.

For the region close to the wall in Fig. 11a,b, and c, the effect of the external fluctuations is not as significant as in the outer layer, but considerable changes can still be observed. The peak at $f^{+} \approx 0.6$ exists in this position very close to the wall at stations $\mathrm{B}$ and $\mathrm{C}$. The resonance mechanism driven by the grid-generated fluctuations can reach the near wall region. There is only a small change in the high frequency spectrum. At station $\mathrm{C}$, spectra are reasonably similar with and without the grid turbulence.

\section{Computational Model Using Fluent}

\subsection{Setup for the simulation conditions}

A numerical simulation was performed using FLUENT 6.0 CFD Code. The RNG $k-\varepsilon$ method in the package was employed to simulate the wall jet flow under the influence of high amplitude external velocity fluctuations. Figure 12 shows a sketch of the 
Fig. 12 Geometry of the boundaries used for FLUENT simulations. Grid generated turbulence was simulated using a sequence of the inlet/outlet jets. $f_{\mathrm{o}}=1 \mathrm{~Hz}$, the grid oscillating frequency

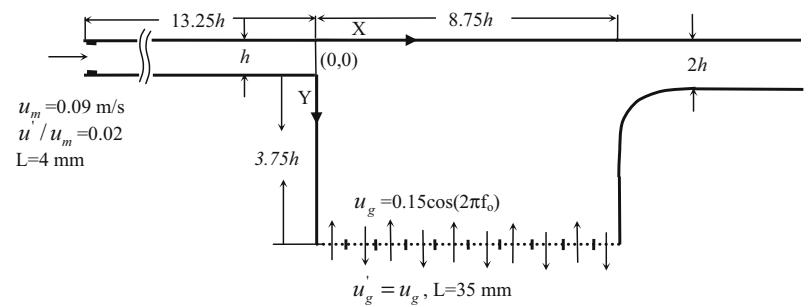

computational domain in which the dimension is equivalent to the experimental setup. Analogous to the experiments, two blocks were set upstream of the channel to trigger the turbulent boundary layer. The triangular meshes were generated using the Gambit software of the FLUENT package. The computation used a wall enhanced treatment that is, there are more grid nodes in the near wall region, to obtain accurate results. The calculated wall jet profiles contained 10 points in the near wall region $y^{+}<10$ and at least 70 points in the rest of the profile.

Simulation of the grid-generated turbulence performed with direct use of a CFD code cannot be done because the turbulent parameterisation in FLUENT or any other standard code is not applicable to the kind of turbulence produced by an oscillating grid. Indeed full numerical simulations (e.g. DNS) have not yet been able to model the variation of turbulent intensity and length scale by a stirrer in a mixing box. Hopfinger and Toly [23] observed that the turbulence generated by the oscillating grid is the appearance of the wakes and jets behind the grid bars. Hence, a set of inlet/outlet jets, as illustrated in Fig. 12, was established to model the high intensity and large scale of the grid generated oscillatory turbulent flow. In this innovative but artificial approach, a periodic array sinusoidal inlet/ outlet jets was located at the mean position of the real grid, $5 h$ from the top wall. The width of each outlet jet was $35 \mathrm{~mm}$. The velocity amplitude of the jets was $0.15 \mathrm{~m} / \mathrm{s}$ according to the moving speed of the grid in experiments at $1 \mathrm{~Hz}$.

The computation starts from the inlet of the inlet channel. According to the velocity profile and turbulence intensity measured behind the upstream meshes using LDA. A uniform inlet velocity at the beginning of the channel was set to $0.09 \mathrm{~m} / \mathrm{s}$. The initial turbulent intensity was set to $2 \%$ of the inlet velocity.

\subsection{Simulation results}

The simulation results of the mean velocity profiles are compared in Fig. 5. For a pure wall jet flow, the model predicts that the mixing layer is thicker than the measured value because the simulations predict a stronger backward circulation in the box. This is inconsistent with the experiment which shows that the external turbulence increases the wall jet thickness and does not induce a significant backward circulation. Regarding the mean flow velocity, the models predict smaller maximum velocities than the experiments, with and without the external turbulence. Using the model of inlet/outlet jets, the external turbulence reduces the maximum velocity. This is in agreement with the experiments.

Figure 6 depicts the non-dimensional form of the predicted velocity profile compared with the observations. When scaled with the upper half-width $y_{1 / 2}^{U}$, with and without the external turbulence the two predicted profiles are self-similar in the upper layer approximately between $y_{1 / 2}^{U}<1$ and $y_{m}$ and also agree well with the measurements. In contrast, using the lower half-width $y_{1 / 2}^{L}$ as the scaling parameter, the two predicted lines collapse to a single line in the lower layer but not in the upper layer. Agreement with the 
experiments is also observed in the lower layer. The FLUENT simulation shows incomplete similarity of the wall jet for the various levels of the free stream turbulence.

The prediction of turbulent fluctuations is displayed in Fig. 9. The simulated profiles without the external turbulence are generally in agreement with the observations. Two local peaks P1 and P2 are formed in the outer and inner layers, respectively. Some minor difference with the experiments can be observed. For example, in the mixing layer P1 decreases with downstream distance. However, the measurements reveal that P1 initially increases, but is approximately the same at stations $\mathrm{C}$ and $\mathrm{D}$. In the inner layer, the profiles obtained from FLUENT show that P2 remains approximately unchanged with the downstream distance. However, the experiments show an increase of P2.

With the generation of the external turbulence, the model successfully gives a significantly stronger turbulent energy in the outer mixing region. The fluctuations decay rapidly with the increase of the distance from the grid. There is reasonable agreement with the experimental turbulence data in the region around P1. Despite good agreement at station $\mathrm{B}$ in the inner layer, the model fails to predict the significant measured increase of the turbulent energy. For example, at station D, the measured value of $\mathrm{P} 2$ increases about three times in comparison to the case without external fluctuations. The simulations only predicted a $15 \%$ increase.

In order to understand whether this discrepancy between the simulation and the measurements is caused by the inadequate modeling of the large scale eddies in the external turbulence, an approximate rapid distortion theory (RDT) model calculation was performed (see Appendix). This shows that intense large scale eddies impacting on the top surface randomly displace the shear layer; the mean height $y / y_{1 / 2}^{U}$ and shear layer thickness increase, and the mean wall shear increases. These effects are consistent with the observed changes.

\section{Conclusions}

An experimental study for the plane turbulent wall jet at the inlet Reynolds number of 2200 under the action of the external turbulence was carried out using LIF flow visualization and LDA measurements. The experiment shows how external velocity fluctuations changes significantly the velocity profile and the energy of fluctuations in the mixing outer layer, causing a rapid growth of the wall jet. The fluctuations also increase in the mixing layer and interact with the flow in the inner layer, which results in an amplification of the turbulence energy in the region of laminar sublayer. Bursting in the mixing layer appears to be stimulated by the external turbulence, which transports energy to the wall region. The skin friction is slightly reduced because the mean thickness of the jet is increased, although the fluctuations slightly compensate for this effect. Spectral analysis shows that a resonance mechanism and the effect of the grid oscillations appear to cause interactions between the external turbulence and shear layer. This results in the energy significantly increasing at the non-dimensional frequency of $f^{+}=0.6$.

CFD simulations using the FLUENT code and a series of small inlet/outlet jets to represent the large scale fluctuations did not model the increase of the wall jet thickness. The model predicts the correct trend in the change of energy transported to the mixing layer, but does not predict the correct level within the shear layer. This result differs from the experimental finding that with $u_{g}^{\prime} / u$ greater than about of 0.1 , the grid generated fluctuations amplify the velocity fluctuations in the inner layer. This incidentally implies that even though there may be a sheltering effect of large scale fluctuations by the outer 
shear layer, there is an increase in smaller scale fluctuations near the wall as a result of nonlinear interactions [6]. A simple statistical/dynamical model, based on rapid distortion concepts, simulates how very large scale eddies can randomly displace the shear layer. This leads to qualitative predictions for the observed effects, and shows that more complex models are needed when the external turbulence contains large intense eddies, as in this experiment.

Acknowledgement We would like to thank Ing R van den Boom for his design of the facility and operating system. This work is supported by FOM (no.99MFS19) and Corus in The Netherlands. We are grateful for useful comments by Dr T. W. D. Peeters and Dr J. Miedema of Corus and Professor A. J. Grass in UCL.

\section{Appendix A}

Simplified model of large scale velocity fluctuations interacting with a jet near a wall

Consider large scale random velocity fluctuations $w(x, y)$ (which are assumed to be two dimensional for simplicity) to have a horizontal scale $l_{x}$ and vertical scale $l_{y}$. Near a wall at $y=0$ these can be approximated by $w=w_{e} y / l_{y}$, where $y \ll l_{x}$, and $w_{e}$ is a random Gaussian variable whose root mean square value is approximately equal to $u^{\prime}$ for the external turbulence [32]. At time $\mathrm{t}=0$, this velocity field now interacts with a weak shear layer with mean velocity $U_{o}(y)$, (where $U(y) \ll\left|w_{o}\right|$ ) whose thickness $l_{j o}$ is small compared with $l_{y}$ (corresponding with our experiments $l_{j o}=y / y_{1 / 2}$ ). For this problem the shear layer is a jet positioned at $y_{j}$, where $y_{j} \ll l_{y}$. The main effect of the turbulence on the jet is (because $l_{x} \gg l_{y}$ ) to move it randomly in the $y$ direction, so that the mean thickness of the jet increases. This is an identical mathematical problem to that of turbulent dispersion, so that for a time less than the integral time scale $T_{L}$, i.e. $T_{L}>t>0$,

$$
U(y, t)=U\left(y_{o}, 0\right)
$$

where $y-y_{o}=t w_{e}\left(1-y / l_{y}\right)$. Thus $U(y, t)$ is now a random variable. Since the displacement $y-y_{o}$ is a random variable (for given $y$ ) the mean value of $U(y, t)$, denoted by $\left\langle U(y, t)>\right.$, is obtained by integrating over all possible values of $y_{o}$ for particles that reach $y$ at time $t$. Thus

$$
<U(y, t)>=\int U\left(y_{o}\right) p\left(y_{o} / y\right) d y_{o}
$$

For a Gaussian large scale velocity field

$$
p\left(y_{o} / y\right)=\frac{1}{\sqrt{2 \pi} y^{\prime}} \exp \left[-\frac{\left(y-y_{o}\right)^{2}}{2 y^{\prime 2}}\right]
$$

where $y^{\prime}$ is the rms displacement, i.e. $y^{\prime}=\sqrt{<\left(y-y_{o}\right)\left(y-y_{o}\right)>}$. So that

$$
\left.y^{\prime}=u^{\prime}\left(y / l_{y}\right) t\right]
$$


For simplicity we take the case where the jet, initially located at $y_{j}$, is very thin, so that

$$
U\left(y_{o}\right)=U_{j} l_{j} \delta\left(y_{o}-y_{j}\right)
$$

where delta is a delta function with unit integral.

Thence combining (4)-(7) it follows that

$$
<U(y, t)>=\frac{1}{\sqrt{2 \pi} y^{\prime}} U_{j} l_{j} \exp \left[-\frac{\left(y-y_{j}\right)^{2}}{2 y^{\prime 2}}\right]
$$

This result shows how the thickness $l_{j}$ becomes $y^{\prime}\left(t, y_{j}\right)$, which increases with time (and in this model is much greater than $l_{j}$, the initial thickness). Because of the effect of the wall which reduces $v$ near $y=0, y^{\prime}$ is greater when $y>y_{j}$ than when $y, y_{j}$. Thus the jet becomes asymmetric, so that its (outer) thickness away from the wall (i.e. $l_{j+}=\int_{y_{j}}^{\infty}\left(y-y_{j}\right)$ $\left.U(y, t) d y / \int_{y_{j}}^{\infty} U(y) d y\right)$ grows more rapidly than the (inner) thickness $l_{j-}$ between $y_{j}$.

Most significantly the height $y_{\max }$ where $\langle U(y)\rangle$ is maximum initially decreases and then as time increases this height increases. In this simplified model there are two maximum downstream. The largest velocity is located at the maximum where $y-y_{j} \approx y_{j} t u^{\prime} / l_{y}$. Note that this complex development shows the sensitivity of the distorted jet profile to the length scale and structure of the external turbulence.

For the experiments in this paper, the length scale $L_{x}$ of the external turbulence is about $3 h=3 l_{j}$, in the notation of the analysis. Thus the approximation of large scale turbulence is appropriate. These results of this statistical, rapid distortion model approximately correspond to the observations, as discussed in Section 4.2.

\section{References}

1. Simonich, J.C., Bradshaw, P.: Effect of free-stream turbulence on heat transfer through a turbulent boundary layer. J. Heat Transfer 100, 671-677 (1978)

2. Kondjoyan, A., Peneau, F., Boisson, H.: Effect of high free stream turbulence on heat transfer between plates and air flows: a review of existing experimental results. Int. J. Therm. Sci. 41, 1-16 (2002)

3. Hunt, J.C.R., Durbin, P.A.: Perturbed vortical layers and shear sheltering. Fluid Dynamics Res. 24, 375404 (1999)

4. Hunt, J.C.R., Eames, I., Westerweel, J.: Mechanics of inhomogeneous turbulence and interfacial layers. J. Fluid Mech. 554, 499-519 (2006)

5. Taylor, G.I.: The spectrum of turbulence. Proc. R. Soc. Lond., A 164, 476-490 (1938)

6. Wu, X., Jacobs, R.G., Hunt, J.C.R., Durbin, P.A.: Simulation of boundary layer transition induced by periodically passing wakes. J. Fluid Mech. 398, 109-1531 (1999)

7. Hunt, J.C.R., Savill, A.M.: Guidelines and criteria for the use of turbulence models in complex flows. In: Hewitt, G.F., Vassilicos, J.C. (eds.) Prediction of Turbulent Flows, pp. 291-343. Cambridge University Press, Cambridge (2005)

8. Blair, M.F.: Influence of free-stream turbulence on turbulent boundary layer heat transfer and mean profile development, Part I - experimental data. J. Heat Transfer 105, 33-40 (1983)

9. Blair, M.F.: Influence of free-stream turbulence on turbulent boundary layer heat transfer and mean profile development, Part II - analysis of results. J. Heat Transfer 105, 41-47 (1983)

10. Hancock, P.E., Bradshaw, P.: The effect of free stream turbulence on turbulent boundary layers. J. Fluid Eng. 105, 284-289 (1983)

11. Maciejewski, P.K., Moffat, R.J.: Heat transfer with very high free-stream turbulence: part I experimental data. J. Heat Transfer 114, 827-833 (1992)

12. Maciejewski, P.K., Moffat, R.J.: Heat transfer with very high free-stream turbulence: part II - analysis of results. J. Heat Transfer 114, 834-839 (1992) 
13. Wood, D.H., Bradshaw, P.: A turbulent mixing layer constrained by a solid surface. Part 2. Measurements in the wall-bounded flow. J. Fluid Mech. 139, 347-361 (1984)

14. Mydlarski, L., Warhaft, Z.: On the onset of high-Reynolds-number grid-generated wind tunnel turbulence. J. Fluid Mech. 320, 331-368 (1996)

15. Thomas, N.H., Simpson, J.E.: Mixing of gravity currents in turbulent surroundings. In: Hunt, J.C.R. (ed.) Turbulence and Diffusion in Stable Environments, Based on the Proceedings of a Conference on Models of Turbulence and Diffusion in Stably Stratified Regions of the Natural Environment, pp. 61-95. Clarendon, Oxford (1986)

16. Eriksson, J.G., Karlsson, R.I., Persson, J.: An experimental study of a two-dimensional plane turbulent wall jet. Exp. Fluids 25, 50-60 (1998)

17. George, W.K., Abrahamsson, H., Eriksson, J., Karlsson, R.I., Lofdahl, L., Wosnik, M.: A similarity theory for the turbulent plane wall jet without external stream. J. Fluid Mech. 425, 367-411 (2000)

18. Tachie, M.F., Balachandar, R., Bergstrom D.J.: Scaling the inner region of turbulent plane wall jets. Exp. Fluids 33, 351-354 (2002)

19. Barenblatt, G.I., Chorin, A.J., Prostokishin, V.M.: The turbulent wall jet: a triple-layered structure and incomplete similarity. Proc. Natl. Acad. Sci. U. S. A. 102, 8850-8853 (2005)

20. Kiya, M., Ohyama, M., Hunt, J.C.R.: Vortex pairs and rings interacting with shear-layer vortices. J. Fluid Mech. 172, 1-15 (1983)

21. Thole, K.A., Bogard, D.G.: High free stream turbulence effects on turbulent boundary layers. J. Fluid Eng. 118, 276-284 (1996)

22. Fernado, H.J.S., De Silva, I.P.D.: Note on secondary flows in oscillating-grid, mixing-box experiments. Phys. Fluids 5, 1849-1851 (1993)

23. Hopfinger, E.J., Toly, J.-A.: Spatially decaying turbulence and its relation to mixing across density interfaces. J. Fluid Mech. 78, 155-175 (1976)

24. Buchave, P., George, W.K., Lumley, J.L.: The measurement of turbulence with the Laser-Doppler Anemometer. Annu. Rev. Fluid Mech. 11, 443-503 (1979)

25. Edwards, R.V.: Report of the special panel on statistical particle bias problems in laser anemometry. J. Fluids Eng. 109, 89-93 (1987)

26. Drain, L.E.: The Laser Doppler Technology. Wiley, New York (1980)

27. Drust, F., Jovanovic, J., Sender, J.: LDA measurement in the near-wall region of a turbulent pipe flow. J. Fluid Mech. 295, 305-335 (1993)

28. Niederschulte, M.A., Adrian, R.J., Hanratty, T.J.: Measurements of turbulent flow in a channel at low Reynolds numbers. Exp. Fluids 9, 222-230 (1990)

29. Head, M.R., Bandyopadhyay, P.: New aspects of turbulent boundary-layer structure. J. Fluid Mech. 107, 297-338 (1981)

30. Brown, G.L., Thomas, A.S.W.: Large structure in a turbulent boundary layer. Phys. Fluids 20, S243S525 (1997)

31. Kim, K.C., Adrian, R.J.: Very large-scale motion in the outer layer. Phys. Fluids 11, 417-422 (1999)

32. Hunt, J.C.R., Graham, J.M.R.: Free stream turbulence near plane boundaries. J. Fluid Mech. 84, 209235 (1978) 